\title{
Projected Range Dependent Tunneling Current of Asymmetric Double Gate MOSFET
}

\author{
Hak Kee Jung \\ Department of Electronic Engineering, Kunsan National University, Korea
}

\begin{tabular}{l} 
Article Info \\
\hline Article history: \\
Received Aug 19, 2015 \\
Revised Nov 8, 2015 \\
Accepted Nov 23, 2015 \\
\hline Keyword: \\
Asymmetric double gate \\
Gaussian function \\
Projected range \\
Thermionic current \\
Tunneling current
\end{tabular}

Article Info

Revised Nov 8, 2015

Accepted Nov 23, 2015

\section{Keyword:}

Gaussian function

Thermionic current

Tunneling current

\begin{abstract}
This study is to analyze the changes of tunneling current according to projected range, a variable of Gaussian function of channel doping function of Asymmetric Double Gate; ADG MOSFET. In MOSFET with channel length below $10 \mathrm{~nm}$, tunneling current occupies a large percentage among off-currents. The increase of tunneling current has a large effect on the characteristics of subthreshold such as threshold voltage movement and the decline of subthreshold swing value, so the accurate analysis of this is being required. To analyze this, potential distribution of series form was obtained using Gaussian distribution function, and using this hermeneutic potential distribution, thermionic emission current and tunneling current making up off-current were obtained. At this point, the effect that the changes of projected range, a variable of Gaussian distribution function, have on the ratio of tunneling current among off-currents was analyzed. As a result, the smaller projected range was, the lower the ratio of tunneling current was. When projected range increased, tunneling current increased largely. Also, it was observed that the value of projected range which the ratio of tunneling current increased changed according to maximum channel doping value, channel length, and channel width.
\end{abstract}

Copyright (C) 2016 Institute of Advanced Engineering and Science. All rights reserved.

\section{Corresponding Author:}

Hak Kee Jung, Department of Electronic Engineering, Kunsan National University, 558 Daehakro, Gunsan, Jeonbuk, Korea, 573-701

Email: hkjung@kunsan.ac.kr

\section{INTRODUCTION}

The largest concerns in semiconductor integrated circuits are high-speed operation and low power consumption. To satisfy these two characteristics, the efforts to improve the way of designing the integrated circuits and to reduce the size of the transistor used in the circuits are being made. However, reduction in the size of transistor shows serious problems in the characteristics of subthreshold such as the decline of subthreshold swing value, threshold voltage movement, and drain induced barrier lowering (DIBL) by short channel effects (SCEs). Especially, the increase of off-current shows the problem with the increase of power consumption by current flow which can't be ignored in the off-state transistor.

The device which has been developed for reducing SCEs is the multiple-gate MOSFET. The multiple-gate MOSFET is the device that the characteristics of subthreshold are improved by producing several gates able to control the current flow in the channel around the channel. The multiple-gate MOSFETs are largely divided into FinFET, double-gate (DG) MOSFET, and cylindrical MOSFET. Among them, DGMOSFET is being studied a lot due to its simple structure. DGMOSFET, as the structure of producing two gates at the top and the bottom, is divided into the symmetric DGMOSFET with the structure same at both sides and asymmetric DGMOSFET (ADGMOSFET) produced with the structure different at each side, and ADGMOSFET of the two is being studied a lot because of the advantage of the increase in the factor able to control the characteristics of the subthreshold [5]. 
Especially, Ding et al. interpreted the subthreshold or something by calculating the hermeneutic potential distribution of ADGMOSFET [6]. Different from Ding et al, this study will use the Gaussian distribution function as the charge distribution function.

Gaussian distribution function is the distribution changed by two variables, which are projected range to define the maximum value and standard projected deviation to determine the width of distribution. Even in the case of having the length of channel below $10 \mathrm{~nm}$ in ADGMOSFET, the increase of off-current is inevitable. Therefore, this study tries to analyze the ratio-change of tunneling current according to projected range, by calculating tunneling current and thermionic emission current comprising off-current of ADGMOSFET.

In chapter 2, the potential distribution of ADGMOSFET and tunneling current model will be explained. In chapter 3, the ratio-change of tunneling current toward projected range calculated by the parameters of the maximum doping concentration, the length of channel, the thickness of channel, and the voltage of gate will be examined. Chapter 4 will be a conclusion.

\section{POTENTIAL DISTRIBUTION AND TUNNELING CURRENT MODELING OF ASYMMETRIC DOUBLE GATE MOSFET}

Schematic sectional diagram of ADGMOSFET was presented in the Figure 1. As Figure 1 indicates, the voltage of top gate $V_{g f}$ and the voltage of bottom gate $V_{g b}$ can be differently biased and, in this case, it can be indicated that the structural parameter able to control the short channel effect increases because the thickness of oxidative film of top and bottom can be differently appointed in each.

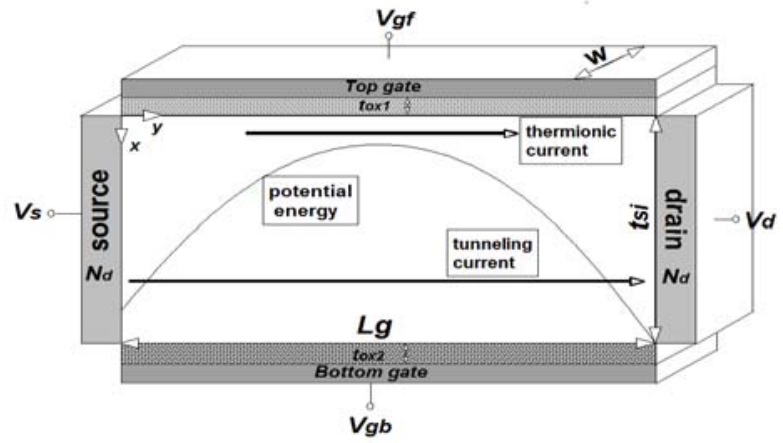

Figure 1. Schematic sectional diagram of asymmetric double gate MOSFET

Hermeneutic potential distribution has been obtained by calculating the Poisson equation of Eq. (1). In this case, Gaussian function, approximate to the experimental value, has been used as the charge distribution function. That is like this.

$$
\frac{\partial^{2} \phi}{\partial x^{2}}+\frac{\partial^{2} \phi}{\partial y^{2}}=\frac{q N_{p}}{\varepsilon_{s i}} \exp \left(-\frac{\left(x-R_{p}\right)^{2}}{2 \sigma_{p}^{2}}\right)
$$

where $\varepsilon_{s i}$ is the permittivity of silicon, $N_{p}$ the maximum doping concentration, $R_{p}$ projected range, and $\sigma_{p}$ the standard projected deviation. In this case, potential distribution obtained by using the boundary condition of Ding et al., is the same as Eq. (2).

$$
\phi(x, y)=V_{s}+\frac{V_{d}}{L_{g}} y+\sum_{n=1}^{\infty}\left[C_{n} e^{k_{n} x}+D_{n} e^{-k_{n} x}+B_{1} \operatorname{erf}\left(\tau+b_{1} / 2\right)+B_{2} \operatorname{erf}\left(\tau+b_{2} / 2\right)\right] \bullet \sin \frac{n \pi y}{L_{g}}
$$

where $V_{s}$ is the source voltage, $V_{d}$ the drain voltage, $n$ a fixed number, $k_{n}=n \pi / L_{g}$, and the other constants are inscribed in the reference [7].

One sixth of electrons moving randomly will head toward drain from source, and, using the number of electrons arriving at $t_{s i} W$ area of drain per unit time, the thermionic emission current is as following. 


$$
I_{\text {ther }}=\frac{q\left(n_{i}^{2} / N_{p}\right) e^{q \phi_{\min }\left(x_{e f f}, y_{\min }\right)} t_{s i} W v_{t h}}{6}
$$

where $x_{\text {eff }}$ is the center of conduction, and $y_{\min }$ is $y$ value that the surface potential gets minimized.

Because, in channel with below 10nm length, the width of energy band from the source to the drain becomes very narrow, tunneling current as well as thermionic emission current can't be ignorable as indicated in Figure 1. Therefore, this study has calculated tunneling current in order to obtain the off current for the ADGMOSFET with the channel length below 10nm. WKB (Wentzel-Kramers-Brillouin) approximation is used to obtain tunneling current. Compared with the approximation of quantum mechanics, WKB approximation is proved to be available one because the difference is ignorable enough [8]. Tunneling current obtained by using WKB approximation is like this.

$$
\begin{aligned}
& I_{\text {tunn }}=\left(\frac{q N_{d} t_{s i} W}{6}\right)\left(\frac{2 T_{t} v_{t h t}}{3}+\frac{T_{l} v_{t h l}}{3}\right) \\
& T_{t, l}=\exp \left[-2 \int_{y_{1}}^{y_{2}}\left|\sqrt{\frac{2 m_{t, l}\left[q \phi\left(x_{\text {eff }}, y\right)-E_{f m}\right]}{\hbar^{2}}}\right| d y\right]
\end{aligned}
$$

where sign $t$ and $l$ mean the values of transversal and longitudinal direction respectively. In this, $v_{t h_{t}}$ and $v_{t h l}$ are thermionic velocities of transversal and longitudinal direction respectively. Total off-current is like this.

$$
I_{\text {tot }}=I_{\text {ther }}+I_{\text {tunn }}
$$

This study will calculate total off-current by using Eq. (5) and then calculate tunneling current ratio according to projected range, the variable of Gaussian distribution function with the parameters such as the length of channel, the thickness of channel, and the doping concentration. Also the change of this ratio will be examined.

\section{CONSIDERATION OF TUNNELING CURRENT OF ADGMOSFET}

Because the validity of Eq. (3) and (4) has already been proved in previously presented thesis [9, 10], this study will calculate thermionic emission current and tunneling current for ADGMOSFET by using Eq. (3) and (4), and consider the change of tunneling current.

At first, the tunneling current for projected range obtained with the parameter of channel length is indicated in Figure 2.

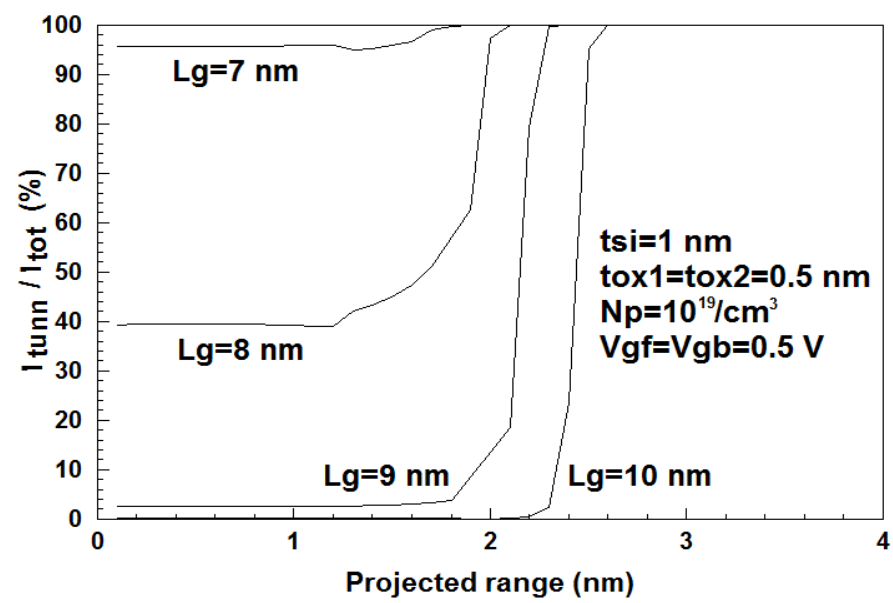

Figure 2. The change of tunneling current ratio for projected range with a parameter of channel length in ADGMOSFET having maximum channel doping concentration of $10^{19} / \mathrm{cm}^{3}$ 
The calculating condition is indicated in the Figure 2. If projected range increases, the ratio of tunneling current among off-current reaches to almost $100 \%$ in the end although it is different according to channel length. It is observed that, as the channel length gets shorter, tunneling current in the smaller extent of projected range reaches to the saturated condition. Especially, in the case of very short channel length around $7 \mathrm{~nm}$, the off-current is composed of $100 \%$ tunneling current regardless of projected range. However, the ratio of tunneling current gets sharply decreased in the smaller extent of projected range if channel length increases, and then it can be observed that the ratio of tunneling current gets saturated as projected range increases. In the case of channel length around $10 \mathrm{~nm}$, the ratio of tunneling current changes sharply from $0 \%$ to $100 \%$ in projected range around $2.3 \mathrm{~nm}$. Like this, the ratio of tunneling current changes sharply according to the channel length.

In the case that channel thickness is the parameter, the change of tunneling current according to the projected range is indicated in Figure 3. As shown in Figure 3a), it can be observed that when channel thickness is big, the ratio of tunneling current greatly increases even in the case projected range is low. However, in the very thin case of channel thickness around $1 \mathrm{~nm}$, the ratio of tunneling current greatly decreases in the small projected range. However, if projected range increases over $2 \mathrm{~nm}$, the ratio of tunneling current increases up to $100 \%$ regardless of channel thickness. In the case of Figure 3b) with $10 \mathrm{~nm}$ of channel length, the ratio of tunneling current increases sharply from $0 \%$ to $100 \%$ according to projected range regardless of channel thickness.

Figure 2 and 3 are the cases that the maximum channel doping concentration is $10^{19} / \mathrm{cm}^{3}$. To observe the change of the ratio of tunneling current according to the change of doping concentration, in the case that channel thickness is the parameter and the maximum doping concentration decreases to $10^{16} / \mathrm{cm}^{3}$, the change of the ratio of tunneling current is shown in Figure 4. Figure 3a) and 4 are the cases that all conditions are same but just the maximum channel doping concentration is different. As Figure 4 shows, it can be observed that the ratio of tunneling current, in projected range around $4 \mathrm{~nm}$, changes from $0 \%$ to $100 \%$ regardless of channel thickness.
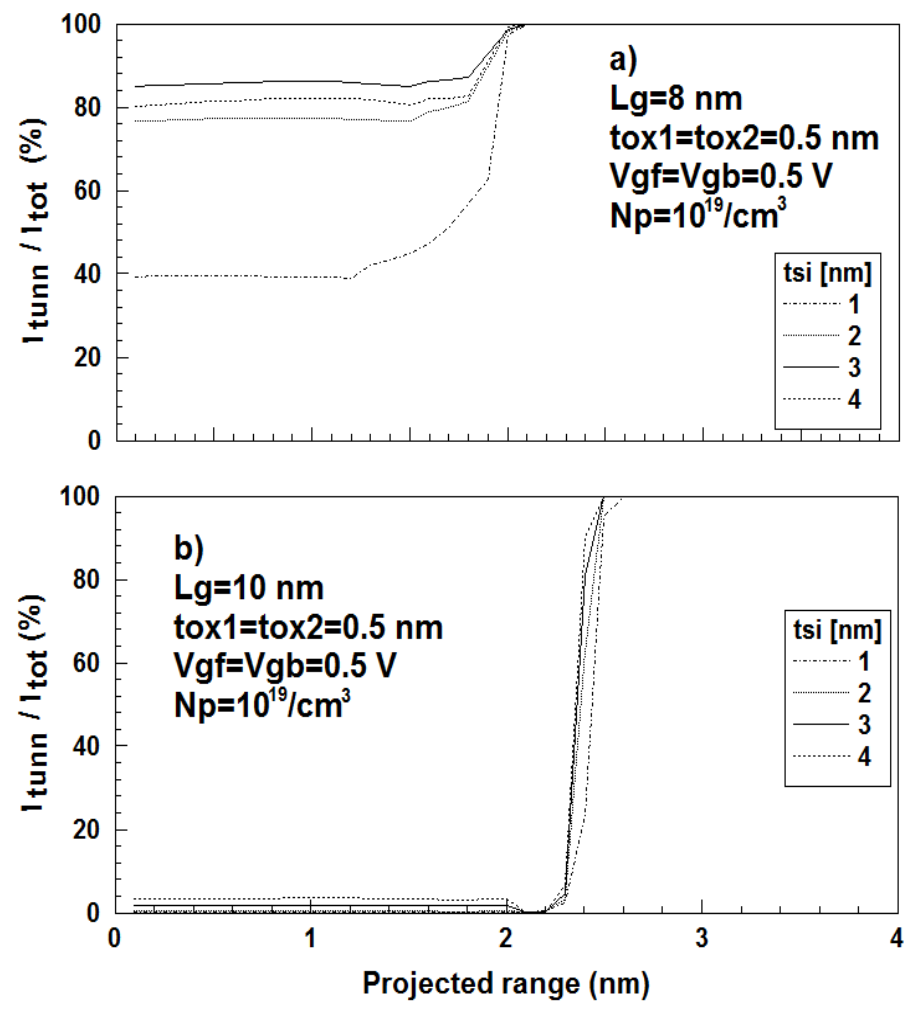

Figure 3. The change of tunneling current ratio for projected range with a parameter of channel thickness in ADGMOSFET in the case of a) $L_{g}=8 \mathrm{~nm}$ and b) $L_{g}=10 \mathrm{~nm}$ 


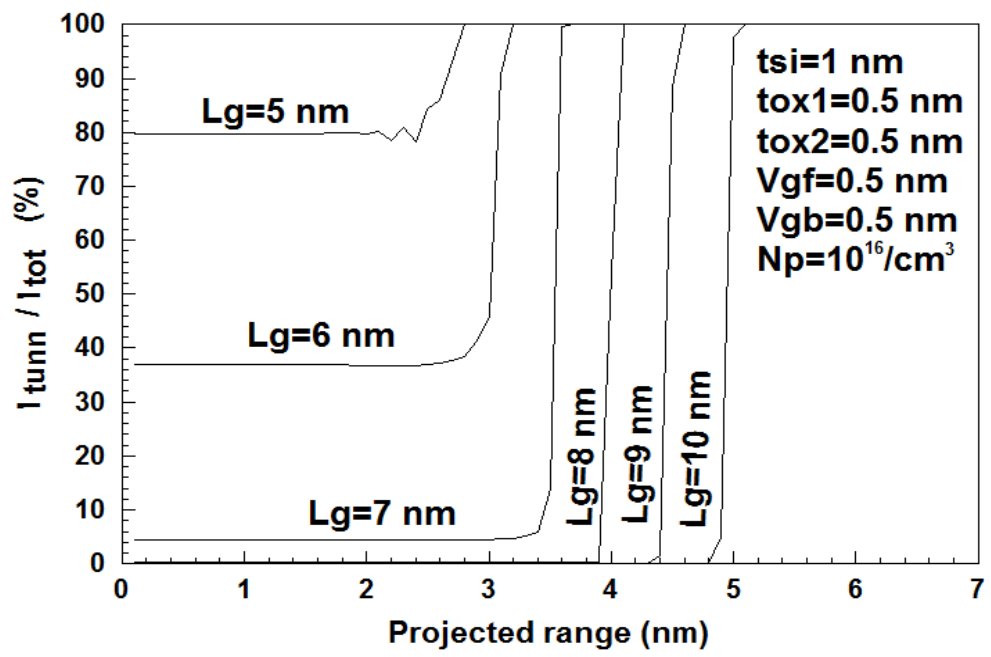

Figure 4. The change of tunneling current ratio for projected range with a parameter of channel thickness in ADGMOSFET having maximum channel doping concentration of $10^{16} / \mathrm{cm}^{3}$

If channel doping concentration decreases, the ratio of tunneling current changes sharply according to projected range rather than channel thickness. In comparison to Figure 3a), if the maximum doping concentration decreases from $10^{19} / \mathrm{cm}^{3}$ to $10^{16} / \mathrm{cm}^{3}$, projected range in which the ratio of tunneling current changes sharply increases from $2 \mathrm{~nm}$ to $4 \mathrm{~nm}$. As Eq. (3) indicates, this is because when the maximum channel doping concentration decreases, thermionic emission current increases and most of the off-current whose projected range reaches up to $4 \mathrm{~nm}$ is occupied by thermionic emission current.

In the case that the maximum channel doping concentration is $10^{16} / \mathrm{cm}^{3}$, the change of the ratio of tunneling current obtained by the parameter of channel length is presented in Figure 5.

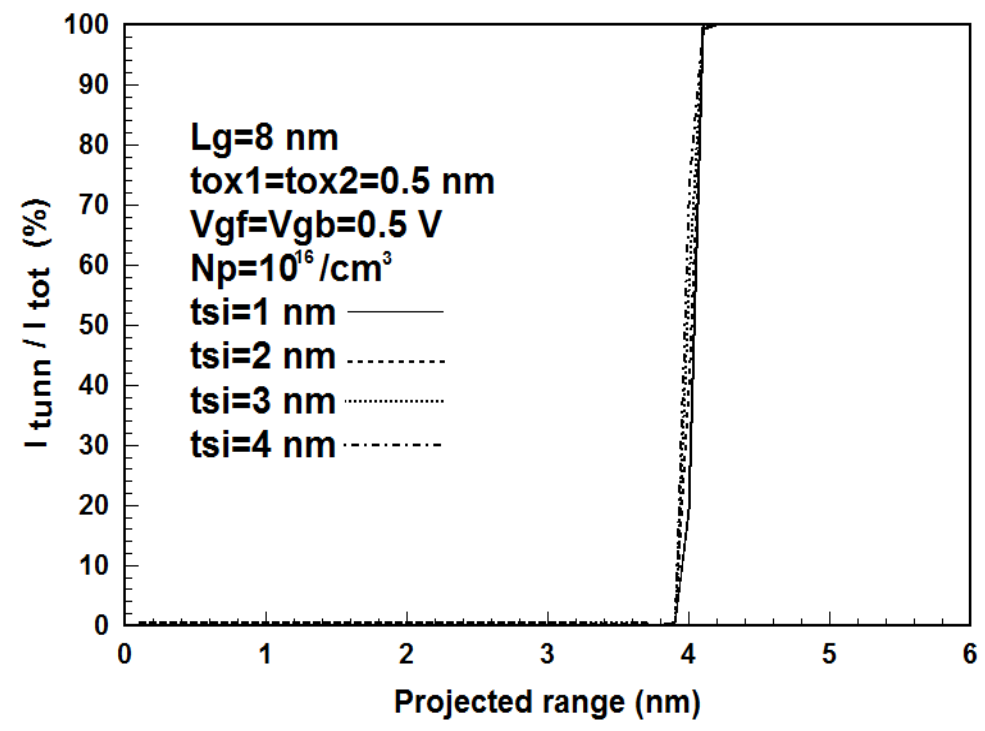

Figure 5. The change of tunneling current ratio for projected range with a parameter of channel length in ADGMOSFET having maximum channel doping concentration of $10^{16} / \mathrm{cm}^{3}$

In comparison of Figure 2, this is just the case that the maximum channel doping concentration decreases from $10^{19} / \mathrm{cm}^{3}$ to $10^{16} / \mathrm{cm}^{3}$. If the maximum channel doping concentration decreases, it can be observed that the ratio of tunneling current decreases greatly because the ratio of thermionic emission increases as previously explained. Especially, if it is compared with the case of channel length of $7 \mathrm{~nm}$, the ratio of tunneling current occupies almost $100 \%$ in the case that maximum channel doping concentration is $10^{19} / \mathrm{cm}^{3}$

Projected Range Dependent Tunneling Current of Asymmetric Double Gate MOSFET(Hak Kee Jung) 
in $2 \mathrm{~nm}$ of projected range as shown in Figure 2, but it remains under the ratio around 5\% when maximum channel doping concentration decreases to $10^{16} / \mathrm{cm}^{3}$ as known in Figure 5. As Figure 5 indicates, in the case that the maximum channel doping concentration is as relatively low as $10^{16} / \mathrm{cm}^{3}$, the value of projected range in which the ratio of tunneling current gets saturated to $100 \%$ changes greatly according to channel length. That is, in the case that the maximum channel doping concentration is low, the change of channel length greatly affects the current because the amount of charge inside the actual channel decreases. That is, in Eq. (3), in the case that $N_{p}$ is small, thermionic current rises greatly, and the ratio of tunneling current decreases greatly in the end.

ADGMOSFET has characteristics that voltage of top and bottom gate can be applied differently. In Figure 6, the change of tunneling current ratio for projected range calculated by the parameter of top gate voltage is presented. At this, bottom gate voltage is fixed at $0.5 \mathrm{~V}$. As Figure 6 indicates, it can be observed that tunneling current ratio decreases if top gate voltage increases. However, if projected range increases over $2 \mathrm{~nm}$, it can be observed that tunneling current ratio increases to $100 \%$ and is saturated regardless of top gate voltage.

If top gate voltage is fixed at $0.5 \mathrm{~V}$ and bottom gate voltage gets changed from $0.2 \mathrm{~V}$ to $0.9 \mathrm{~V}$, the same graph can also be obtained. That is, it can be found that tunneling current ratio shows symmetrical change for top and bottom gate voltage. As for the change of tunneling current in projected range below 2 $\mathrm{nm}$, it shows great change between $0.5 \mathrm{~V}$ and $0.7 \mathrm{~V}$ of top gate voltage. That is, it can be found that tunneling current ratio shows great change in the case that top and bottom gate voltage are similar.

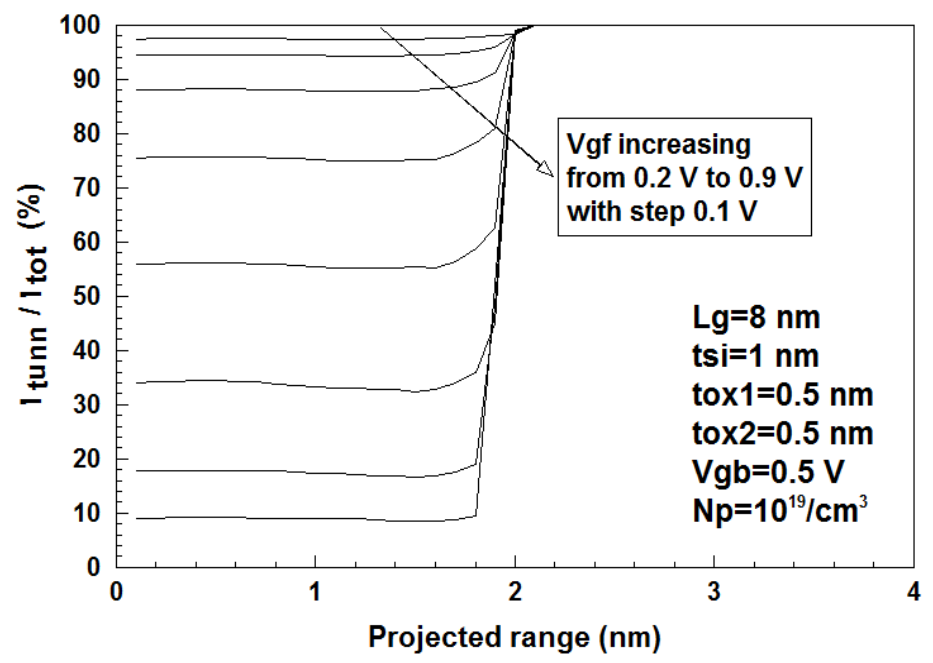

Figure 6. The change of tunneling current ratio for projected range with a parameter of top gate voltage in ADGMOSFET having maximum channel doping concentration of $10^{19} / \mathrm{cm}^{3}$

\section{CONCLUSION}

This study analyzed the change of tunneling current according to projected range which is the variable of the Gaussian function that is channel doping function of ADGMSFET. The ratio change of thermionic emission current and tunneling current which is composing off-current in ADGMOSFET with channel length below $10 \mathrm{~nm}$ was observed according to projected range. At this point, the change of channel size like channel length and channel thickness, of the maximum doping concentration, and of top gate voltage were used as parameters.

As a result, it was found that tunneling current ratio was low as projected range was small, and that tunneling current increased greatly as projected range increased. However, the tendency of change was greatly differentiated according to parameters.

That is, in the case of $10^{19} / \mathrm{cm}^{3}$ of the maximum channel doping concentration, when channel length was as short as $7 \mathrm{~nm}$, almost $100 \%$ of tunneling current composed off-current regardless of projected range. However, if the maximum channel doping concentration decreased to $10^{16} / \mathrm{cm}^{3}$, only around $5 \%$ of off-current, when channel length was $7 \mathrm{~nm}$, was composed of tunneling current due to the increase of thermionic emission current. Especially, projected range in which the tunneling current ratio changed sharply showed great difference according to channel length, 
channel thickness, and the maximum doping concentration. In addition, it was observed that current ratio decreased greatly when top gate voltage increased, and that tunneling current ratio was also saturated to $100 \%$ when projected range increased. Because projected range has a great effect on tunneling current ratio like this, it should be heeded in designing ADGMOSFET.

\section{REFERENCES}

[1] S.M. Lee and J.T. Park, "Electrical properties of nanoscale junctionless p-channel MugFET at cryogenic temperature”, J. Korea Inst. Inf. Commun. Eng., vol. 17, no. 8, pp. 1885-1890, 2013.

[2] W. Chang, L. Cin and W. Yeh, "Impact of Fin Width and Back Bias Under Hot Carrier Injection on Double-Gate FinFETs”, IEEE Trans. on Device \& Materials Reliability, vol. 15, no. 1, pp. 86-89, 2015.

[3] S. Jandhyala and S. Mahapatra,’'Inclusion of body doping in compact models for fully-depleted common double gate MOSFET adapted to gate-oxide thickness asymmetry", Electronics Letters, vol. 48, no. 13, pp. 794-795, 2013.

[4] C.H. Suh,"'Two-Dimensional Analytical Model for Deriving the Threshold Voltage of a Short Channel Fully Depleted Cylindrical/Surrounding Gate MOSFET”, J. of Semiconductor and Science, vol. 11, no. 2, pp. 111-120, 2011.

[5] S. Mohammadi, A. Afzali-Kusha and S. Mohammadi, "Compact modeling of short-channel effects in symmetric and asymmetric 3-T/4-T double gate MOSFETs”, Microelectronics Reliability, vol. 51, pp. 543-549, 2011.

[6] Z. Ding, G. Hu, J. Gu, R. Liu, L. Wang and T. Tang, "An analytical model for channel potential and subthreshold swing of the symmetric and asymmetric double-gate MOSFETs”, Microelectronics J., vol. 42, pp. 515-519, 2011.

[7] Hakkee Jung, "Analysis for Potential Distribution of Asymmetric Double Gate MOSFET Using Series Function”, J. of KIICE, vol. 17, no. 11, pp. 2621-2626. 2013.

[8] M. Stadele, "Influence of source-drain tunneling on the subthreshold behavior of sub-10 nm double gate MOSFETs", in Proc. Eur. Solid-State Device Research Conf.(ESSDERC), Florence, Italy, pp. 135-138, 2002.

[9] H.K. Jung and O.S. Kwon, "Analysis of Channel Dimension Dependent Threshold Voltage for Asymmetric DGMOSFET”, Information, vol. 17, no. 11(B), pp. 5879-5884, 2014.

[10] H.K. Jung and D. Sima, "Analysis of Subthreshold Carrier Transport for Ultimate DGMOSFET", IEEE Trans. on Electron Devices, vol. 53, no. 4, pp. 685-691, 2006.

\section{BIOGRAPHY OF AUTHOR}

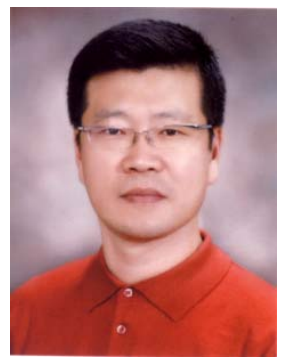

Prof. Hak Kee Jung received the B.S. degree from Ajou University, Korea, in 1983, the M.S. and Ph.D. degrees from Yonsei University, Seoul, Korea, in 1985, 1990, respectively, all in electronic engineering. In 1990, he joined Kunsan National University, Chonbuk, Korea, where he is currently a Professor in department of electronic engineering. From 1995 to 1995, he held a research position with the Electronic Engineering Department, Osaka University, Osaka, Japan. From 2004 to 2005, he was with the School of Microelectronic Engineering, Griffith University, Nathan, QLD, Australia. His research interests include semiconductor device physics and device modeling with a strong emphasis on quantum transport and Monte Carlo simulations. 